Research Paper

\title{
Differential Aspects of Natural and Morphine Reward-related Behaviors in Conditioned Place Preference Paradigm
}

\author{
Shole Jamali $^{1}$ (D), Mahdi Aliyari Shoorehdeli² (D), Mohammad Reza Daliri ${ }^{3,4}$ (D), Abbas Haghparast ${ }^{*}$ (D) \\ 1. Neuroscience Research Center, School of Medicine, Shahid Beheshti University of Medical Sciences, Tehran, Iran. \\ 2. Department of Mechatronics, School of Electrical Engineering, K. N. Toosi University of Technology, Tehran, Iran. \\ 3. School of Cognitive Sciences, Institute for Research in Fundamental Sciences, Tehran, Iran. \\ 4. Department of Biomedical Engineering, School of Electrical Engineering, Iran University of Science and Technology, Tehran, Iran.
}

\begin{tabular}{|c|c|}
\hline $\begin{array}{l}\text { Use your devivec to scan } \\
\text { and read the article online }\end{array}$ & dtation Jamali, S., Aliyari Shoorehdeli, M., Daliri, M. R., and Haghparast, A. (2022). Differential Aspects of \\
\hline 口itista & $\begin{array}{l}\text { Natural and Morphine Reward-related Behaviors in Conditioned Place Preference Paradigm. Basic and Clinical Neuro- } \\
\text { science, 13(5), 731-744. http://dx.doi.org/10.32598/bcn.2021.3071.1 }\end{array}$ \\
\hline a & dol'http://dx.doi.org/10.32598/bcn.2021.3071.1 \\
\hline
\end{tabular}

\section{Article info:}

Received: 25 Nov 2020

First Revision: 27 Jan 2021

Accepted: 28 Jan 2021

Available Online: 01 Sep 2022

Keywords:

Natural reward, Morphine reward, Food deprivation,

Food restriction, Conditioned place preference, Rat

\begin{abstract}
A B S T RA C T
Introduction: Natural rewards are essential for survival. However, drug-seeking behaviors can be maladaptive and endanger survival. The present study was conducted to enhance our understanding of how animals respond to food and morphine as natural and drug rewards, respectively, in a conditioned place preference (CPP) paradigm.

Methods: We designed a protocol to induce food CPP and compare it as a natural reward with morphine CPP in rats. The protocol for reward induction in both groups (foods and morphine) consisted of three phases: pre-test, conditioning, and post-test. In morphine groups, we injected morphine as a reward $(5 \mathrm{mg} / \mathrm{kg}, \mathrm{SC})$. To induce natural reward, we used two different protocols. In the first one, the rats were deprived of food for $24 \mathrm{~h}$. In the other method, the rats were restricted to food for 14 days. During the conditioning period, the animals received daily chow, biscuits, or popcorn as a reward inducer.

Results: Results revealed that CPP was not induced in food-deprived rats. A combination of food restriction (as a facilitator) and a biscuit or popcorn-induced reward using CPP. In contrast, food deprivation did not facilitate food CPP in response to regular food. Interestingly the CPP score of the group which received biscuits during a 7-day conditioning period was more than that of the morphine group.
\end{abstract}

Conclusion: In conclusion, food restriction could be a better protocol than food deprivation to facilitate food reward. 


\section{Highlights}

- Consumption of regular did not induced conditioned place preference in food deprived rats.

- Palatable food induced the conditioned place preference in food-restricted rats.

- Food restriction was a better facilitator than food deprivation for induction of food/natural reward.

- Specific food as compared with morphine significantly induced the conditioned place preference in the rats.

\section{Plain Language Summary}

In today's society, addiction is a growing problem, which is not only restricted to drugs, but also to foods, sex, and shopping as well. On the other hand, to maintain a healthy lifestyle, we must also enjoy natural rewards. In drug addiction treatment, one of the goals is to maintain the natural response. This kind of treatment requires an understanding of the brain reward circuits underlying drug and natural rewards. We examined inducing morphine$\mathrm{CPP}$ as a drug reward and designed different conditioned place preference behavior (CPP) paradigms for inducing food reward as a natural reward model to compare drug-induced and natural-induced CPP behavior.

\section{Introduction}

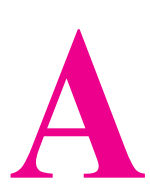

ddiction is a severe public health problem that is not just limited to the abuse of drugs such as morphine, cocaine, and methamphetamine but is also associated with compulsive behavior related to eating food, gambling, sex, and shopping (LewStarowicz, et al., 2020).

Drug and non-drug addiction targets the reward system (Olsen, 2011). One of the non-drug (natural) cues, i.e., food, especially palatable food, could induce reward and affect its circuit. For the first time, "food addiction" was presented in the literature as a term in the 1950s. While many studies have focused on drug addiction, few reports have been published on food addiction (Krashes \& Kravitz, 2014). Morphine is an exogenous opioid used therapeutically to modulate severe, acute, or chronic pain (Rosenblum et al., 2008). However, opioid misuse is a major problem in the world (Strang et al., 2020).

Several areas in the brain are involved in reward-related behaviors, including the Ventral Tegmental Area (VTA), Nucleus Accumbens (NAc), Hippocampus (HIP), prefrontal cortex (PFC), hypothalamus, and amygdala (Lee et al., 2020; $\mathrm{Ng}$ et al., 2013). Induced changes in the reward system's activity are related to prevalent disorders such as addiction and obesity. Thus, understanding the reward-associated behaviors and mechanisms is essential for treating these disorders (Domingo-Rodriguez et al., 2020; Grosshans et al., 2011; Hogarth, 2020; Inbar et al., 2020). The growth of obesity has become a severe problem that affects many countries worldwide. An increase in food reward and consumption can lead to obesity (Leigh \& Morris, 2018; Stice et al., 2013).

The circuit and mechanisms involved in food reward (as a natural reward) and compulsive eating behavior may be similar to those that induce compulsion toward drugs (Krashes \& Kravitz, 2014). Studies suggest similarities between behavioral and neurochemical characteristics of drug addiction and food addiction (Allen et al., 2012; Avena et al., 2009).

On the other hand, treatment of addiction influences natural reward behaviors. It seems that abused drugs and natural rewards both use the same reward circuit (Lau et al., 2006), which is considered a problem in addiction treatment because survival depends on natural rewards such as food and sex. Disrupting the natural reward system could induce anxiety, depression, and other reward-related disorders (Saper et al., 2002). It has been proven that the ability to follow food and water rewards is crucial for animal survival. The critical question is, "What is the difference between naturaland morphine-reward-related behavior in Conditioned Place Preference (CPP)?" To answer this question, we investigated and compared the animals' reward related behavior in morphine- and food-induced CPP.

There are different protocols for the induction of food reward. Food deprivation or food restriction before animal conditioning to food is mostly used in this regard (Toth \& Gardiner, 2000). The challenge of using depri- 
vation and restriction as a motivational stimulator for behavioral training is to find the sweet spot between the severity of the imposed deprivation and restriction schedule and the need for motivated learning or performance. Therefore, one aspect of this experimental study revolves around whether food restriction or deprivation could be a facilitator to food reward induction or not.

Food deprivation and food restriction procedures are necessary parts of various protocols in behavioral studies in neuroscience. They cover protocols for feeding behavior, impulse control, and reward-aversive behavior (Carlini et al., 2008; Conrad, 2010; Ouyang et al., 2017; Williams et al., 2020). "Deprivation" and "restriction" are different terms and concepts. In food reward (as a natural reward model), studies on the deprivation or restriction procedure are not only essential but the type of food that animals receive as the reward is also important (Sampey et al., 2011). There is no single standard food deprivation or restriction protocol to facilitate food reward (Prescott et al., 2010).

Conditioned place preference, as one of the most popular tests, is increasingly used to investigate the rewardrelated effects of drugs and non-drug treatments in animals. In this study, we also designed a CPP paradigm to answer our questions (Marion-Poll et al., 2019; Shirazy et al., 2020).

Therefore, the current study examines the trend of food deprivation and food restriction to induce food reward as a natural reward model. Furthermore, we compare the reward-related behavior of morphine (as a drug reward) with a food reward (as a natural reward model).

\section{Materials and Methods}

Study animals

All subjects $(\mathrm{n}=103)$ were adult male Wistar rats (Pasteur Institute, Tehran, Iran) weighing 220-270g at the start of the experiment. The rats were housed in a $12 / 12 \mathrm{~h}$ light/dark cycle at a temperature of $25^{\circ} \mathrm{C} \pm 2^{\circ} \mathrm{C}$ and humidity of $55 \% \pm 10 \%$ in a controlled central animal facility. All subjects were randomized in groups of two per cage and housed with bedding and free access to water. Experimental procedures were approved by the Ethics Committee of Shahid Beheshti University of Medical Sciences (IR.SBMU.SM.REC.1395.373), Tehran, Iran. All protocols followed the internationally accepted principles for the Care and Use of Laboratory Animals (National Institutes of Health [NIH] publication No. 80-23, revised in 1996).

\section{Drugs}

Morphine sulfate (Tehran, Iran) was dissolved in physiological saline $(0.9 \% \mathrm{NaCl})$ and administered by the subcutaneous (SC) route at the dose of $5 \mathrm{mg} / \mathrm{kg}$ in the conditioning phase.

\section{Food deprivation and food restriction}

Ad libitum-fed subjects had free access to standard lab chow pellets. Food-deprived animals had access to pellets except for $24 \mathrm{~h}$ before the beginning conditioning phase (Food was withdrawn from all subjects $24 \mathrm{~h}$ before the beginning of the conditioning). Food-restricted rats followed a feeding regimen (Carr, Kim, \& de Vaca, 2000; De Vaca \& Carr, 1998; Toth \& Gardiner, 2000) in which they received $50 \%$ of ad libitum chow intake (11g) daily ( $22 \mathrm{~g}$ each cage) until body weight was reduced by $15 \%-20 \%$ (after 14 days). The palatable food (popcorn or biscuit) or regular chow was considered the reward (Ong \& Muhlhausler, 2011). Each rat received $17 \mathrm{~g}$ of food (daily pellet + reward food) for maintaining the body weight during the experiment (or until the end).

After an initial body weight measurement and a pretest, the animals experienced $24 \mathrm{~h}$ food deprivation to facilitate the food reward. They had no access to food. After the deprivation period, animals were weighed. Furthermore, the percentage of body-weight loss, considering the initial weight, was calculated.

To investigate the weight loss trend in animals during the food restriction (FR) period, they were weighed before applying FR (day 0). Subsequently, their weight was recorded on the following checkpoints of days 2 , $4,6,8,10,12$, and 13 . If any significant weight loss happened to an animal observed between any of the specific weight-measuring checkpoints and its previous checkpoint, the animal would have to be excluded from the study and fed with a regular diet to compensate for the excessive weight loss.

\section{Conditioning place preference paradigm}

The experiments were performed in a three-compartment Plexiglas CPP apparatus. The CPP apparatus is a form of classical conditioning used to evaluate motivational properties, such as rewarding or aversive effects of reward-related cues in animals. Two equal-sized compartments (as the main chambers) for the conditioning session, isolated by a guillotine door, lead into the third part, known as the null part $(30 \times 15 \times 40 \mathrm{~cm})$. The floor texture (smooth or rough) and wall-striped 
pattern made the two main compartments different. One of the compartments' walls is striped horizontally, and the other compartment wall is striped vertically. The rats' behavior was monitored through a $3 \mathrm{CCD}$ camera (Panasonic, Japan) held directly above the apparatus. Data were analyzed by EthoVision software (v. 7), a video tracking system for automating behavioral experiments (Noldus Information Technology, the Netherlands). The distance traveled and time spent in each compartment were recorded. The CPP scores were calculated by subtracting the time spent in the non-drug or food-paired compartment from the time spent in the morphine- or food-paired compartment for morphine- and food-CPP scores, respectively. The total distance traveled (in $\mathrm{cm}$ ) was considered as the index of locomotor activity for each animal.

The CPP procedures of all groups consisted of three phases: habituation and pre-conditioning, conditioning, and post-conditioning. Each animal experienced two daily 45-minute sessions during conditioning days.

\section{Habituation and pre-conditioning phase}

Rats were handled at least four days before initiating behavioral testing and habituated for $10 \mathrm{~min}$ in the CPP box one day before pre-conditioning day. Each animal was separately placed in the null compartment. The door was removed, and the rats could move freely in the three compartments for $10 \mathrm{~min}$. The time they spent and locomotor activity in each compartment was calculated. Each animal that spent more than $70 \%$ of total time in either compartment was considered to have initial bias and was excluded from the study (6 in total) (Pourhamzeh et al., 2019). This procedure was performed on all animals.

\section{Experiment 1: morphine conditioning}

On the first day of the conditioning phase, each animal received morphine $(5 \mathrm{mg} / \mathrm{kg}$, SC) in the morning and was immediately confined to the morphine-paired chamber for $45 \mathrm{~min}$; about sixh later, the animals were injected with saline as a vehicle, $(1 \mathrm{~mL} / \mathrm{kg}, \mathrm{SC})$ and were placed in the assigned compartment as a saline-paired compartment for $45 \mathrm{~min}$. On an alternate day, morphine and saline injections time were arranged in a counterbalanced manner. The third day of conditioning was the same as the first day. During this phase, access to other chambers of the CPP box was blocked.

\section{Experiment 2: Food conditioning after deprivation}

To facilitate food reward in rats, after the first bodyweight measurement, the animals were examined for the pre-test phase and then underwent food deprivation while housed in groups of two per cage. After $24 \mathrm{~h}$ of food deprivation, the deprived animals were divided randomly into three groups in which each group experienced 3 days of conditioning period (FD-3d-pellet group), five days of conditioning (FD-5d-pellet group), or 7 days of conditioning (FD-7d-pellet group), while receiving commercial rodent pellet (regular food) as a reward.

Regarding the food-deprived animals that experienced 3-day conditioning (FD-3d-Pellet), the only difference between this and the morphine group was the reward (pellet vs morphine). During the conditioning days, the animals had access to pellets as a reward cue in the food (reward)-paired compartment for 45 min while they were conditioned with nothing in the opposite compartment (Duan et al., 2016) for $45 \mathrm{~min}$ in alternate session. In the food-paired compartment, animals received pellets $(6 \mathrm{~g})$ (Figure $1 \mathrm{~A})$. At the end of the food session, the residual food was removed, collected from the food compartment, and weighed to calculate the amount of food consumed. At the end of each day, the residual food of 6-g reward food plus an 11-g regular pellet was placed in the home cage to maintain the rat's weight until the end of the experiment. Furthermore, on pre- and post-conditioning days, food (as a reward) was absent for all animals.

Regarding the food-deprived animals that experienced 5-day conditioning (FD-5d-Pellet), the procedure was similar to that experienced by the FD-3d-pellet group. The only difference was the conditioning phase duration (5 days: 10 sessions) (Figure 1B).

Regarding the food-deprived animals that experienced 7-day conditioning (FD-7d-Pellet), the procedure was similar to that experienced by the FD-3d-pellet group, and the difference was the number of conditioning days (7 days: 14 sessions) (Figure 1C).

\section{Food conditioning after restriction}

To induce food reward in rats, after the first bodyweight (W0) measurement, they were restricted to food to a target of $80 \%-85 \%$ of their initial body weight. After 14 days of food restriction, the restricted animals were divided randomly into three groups that experienced 3,5 , or 7 days of conditioning, respectively, while receiving palatable food, including biscuit (B) 


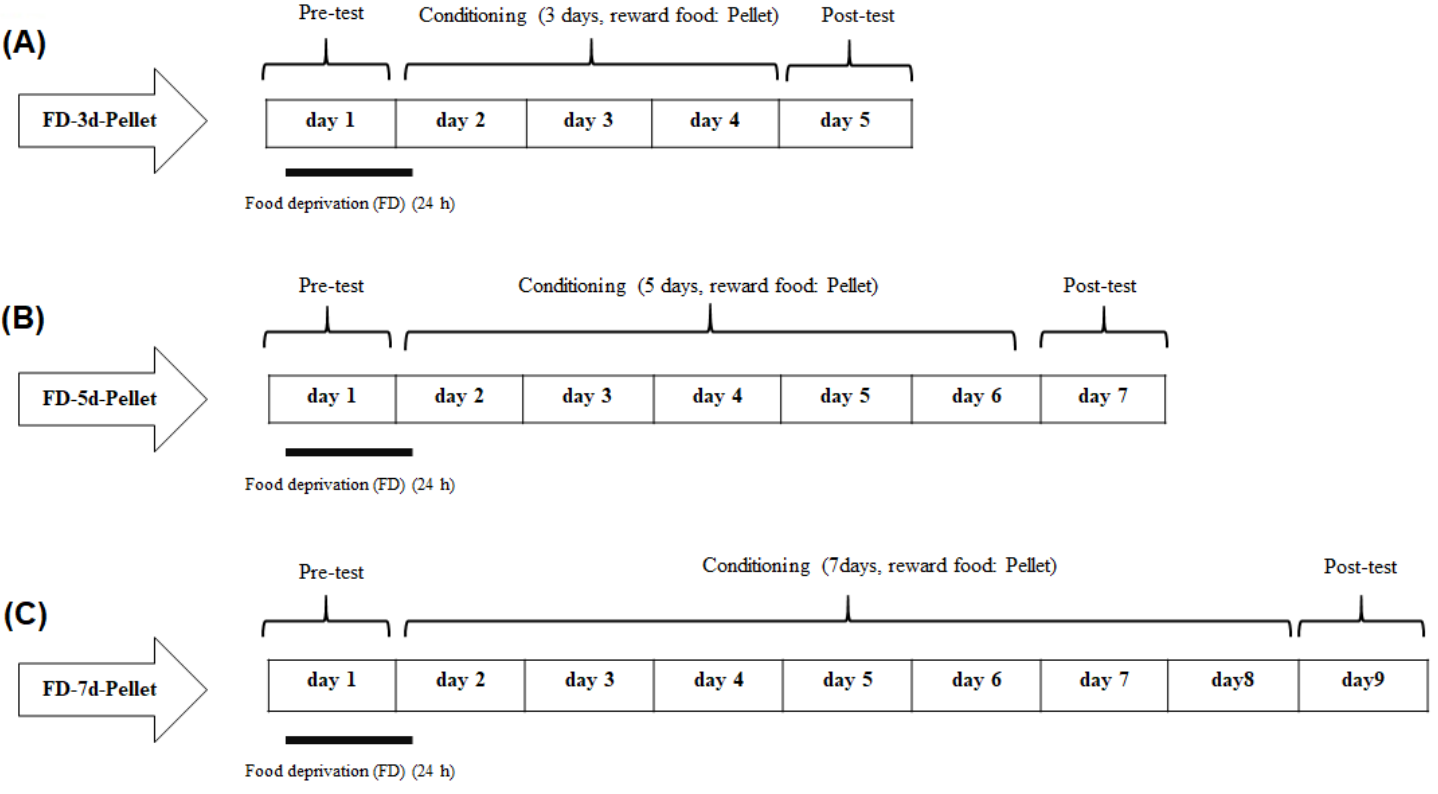

Figure 1. Experimental design for inducing food Conditioned Place Preference (CPP) in deprived animals

NEUROSCIENCE

They received regular chow during 3, 5, or 7 conditioning days.

or popcorn $(\mathrm{P})$, as the reward cues during conditioning phase (Figure 2 parts A, B, and C).

When the food-restricted animals received biscuits as a reward, after the pre-test, the animals were divided into three groups. On the second day of the experiment, the first day morning session of the conditioning period, the animals received $6 \mathrm{~g}$ biscuit as the reward in the mid- dle of the reward compartment, and $6 \mathrm{~h}$ later, they were placed into the no-reward compartment with no food; each session lasted $45 \mathrm{~min}$. On the following days, biscuit and no-food session times were arranged in a counterbalanced manner over the conditioning period. The difference between these three groups was the duration of the conditioning phase. The FR-3d-B group experienced food restriction protocol before starting the con-

(A)
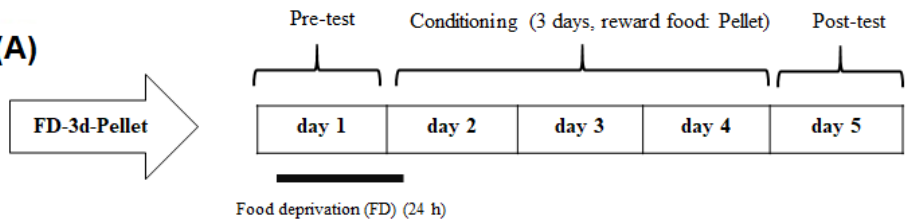

(B)
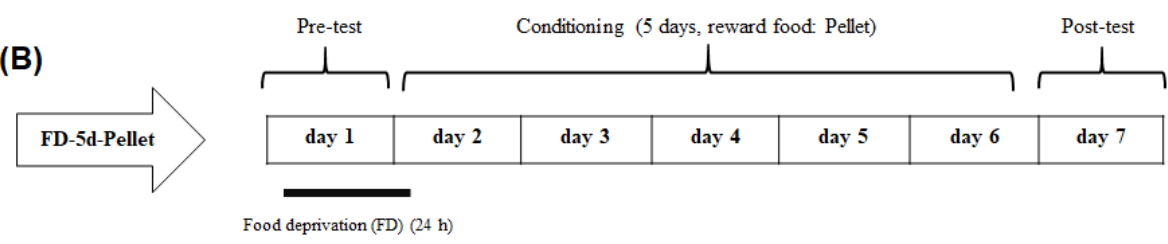

(C)

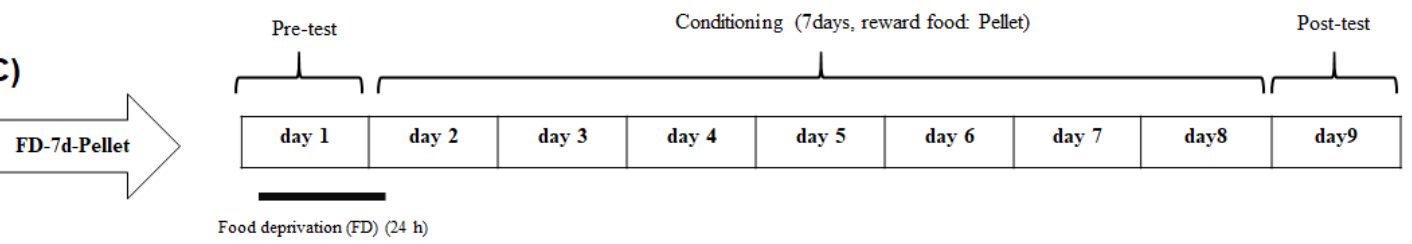

Figure 2. Experimental design of inducing food conditioned place preference (CPP) in restricted animals

They experienced palatable food (biscuits or popcorn) during 3, 5, or 7 conditioning days. 
ditioning phase and received biscuits (B) as the reward during 3 days of conditioning. The FR-5d-B group also underwent a food restriction protocol and was awarded by biscuit during 5 conditioning days. In FR-7d-B, food-restricted animals got biscuits for 7 conditioning days (Figure 2 parts A, B, and C). In this subset, as previously mentioned during the CPP test (pre-test, conditioning phase, and post-test), all animals received $17 \mathrm{~g}$ of food per day ( $6 \mathrm{~g}$ of favorite food [reward] $+11 \mathrm{~g}$ of regular food). In the pre-test and post-test, the animals did not receive any food reward.

When the food-restricted animals received popcorn as a reward, all procedures were similar to the previous section except for the type of food reward. The food-restricted animals received popcorn as a reward instead of a biscuit. After restriction and pre-test, animals were divided into three groups in which they experienced three, five, or seven days of conditioning (FR-3d-P, FR-5d-P, and FR-7d-P, respectively), while receiving popcorn as the reward in food-conditioning sessions and they did not get food in the unrewarded compartment.

\section{Post-test}

Twenty-four hours after the last conditioning day, the animals were examined for CPP, similar to the pre-test. The rats were placed into the null chamber with free access throughout the apparatus for $10 \mathrm{~min}$. The CPP scores were calculated, and the traveled distances were recorded.

\section{Statistical analysis}

The data are expressed as mean \pm SEM, and the Gaussian distribution was tested using the KolmogorovSmirnov normality test. The data were processed by the commercially available software GraphPad Prism (version 5.0). To compare the CPP scores between the pre-test and post-test of each group, we used the paired $t$ test. The 1-way or 2-way ANOVA followed by Tukey's or Bonferroni multiple comparison tests were used to compare the calculated CPP score of post-tests between groups. Also, we used repeated measures 2-way ANOVA followed by Bonferroni to compare the weight loss between the considered times during the food restriction days. The 1-way ANOVA, followed by Tukey multiple comparison test, was used to compare the distance traveled between groups. Results were statistically considered significant when $\mathrm{P}<0.05$.

\section{Results}

\section{Body-weight loss during the restriction period}

To induce food reward, the animals were deprived or restricted from food. FD-3d-pellet, FD-5d-pellet, and FD7-pellet groups were deprived of food for $24 \mathrm{~h}$ before the conditioning phase. The FR-3d-B, FR-5d-B, FR-7d-B, FR-3d-P, FR-5d-P, and FR-7d-P groups were restricted to $50 \%$ of their daily food before the conditioning period for two weeks. During the food-restriction period, the

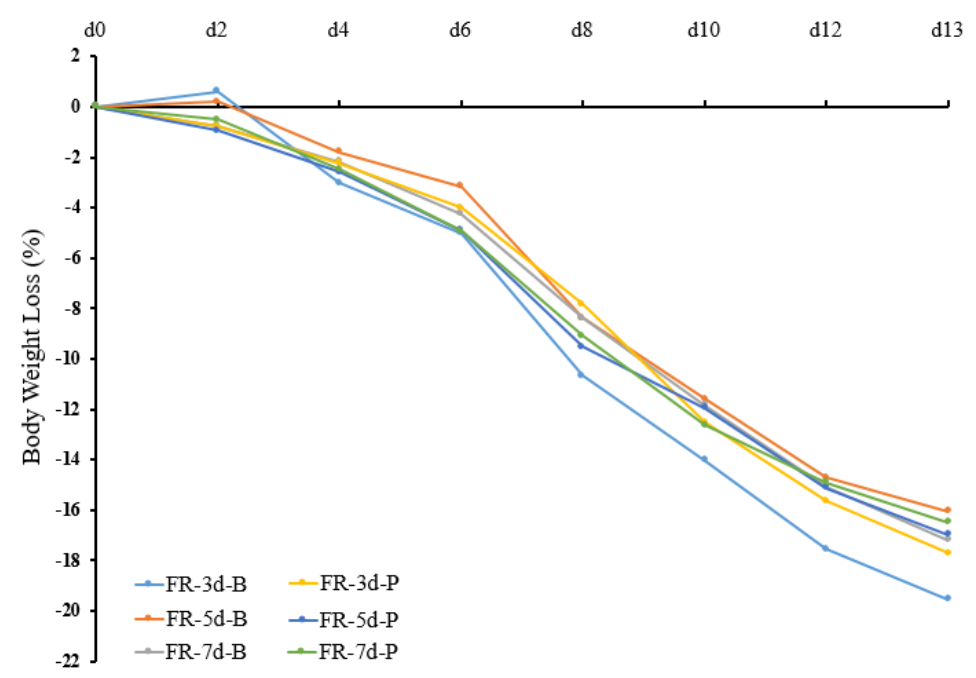

Figure 3. The trend of body-weight loss in rats

Effect of food restriction on body weight loss percentage (WL\%) in restricted rats. Expressed is the mean body weight loss (\%) of each checkpoint concerning an animal's body weight loss at the onset of the food restriction period $(\mathrm{d} 0)(\mathrm{d}=$ day). The checkpoints were $\mathrm{d} 0, \mathrm{~d} 2, \mathrm{~d} 4, \mathrm{~d} 6, \mathrm{~d} 8, \mathrm{~d} 10, \mathrm{~d} 12$, and $\mathrm{d} 13$. There is no significant difference between the weight loss (WL\%) of two consecutive checkpoints in each group. 

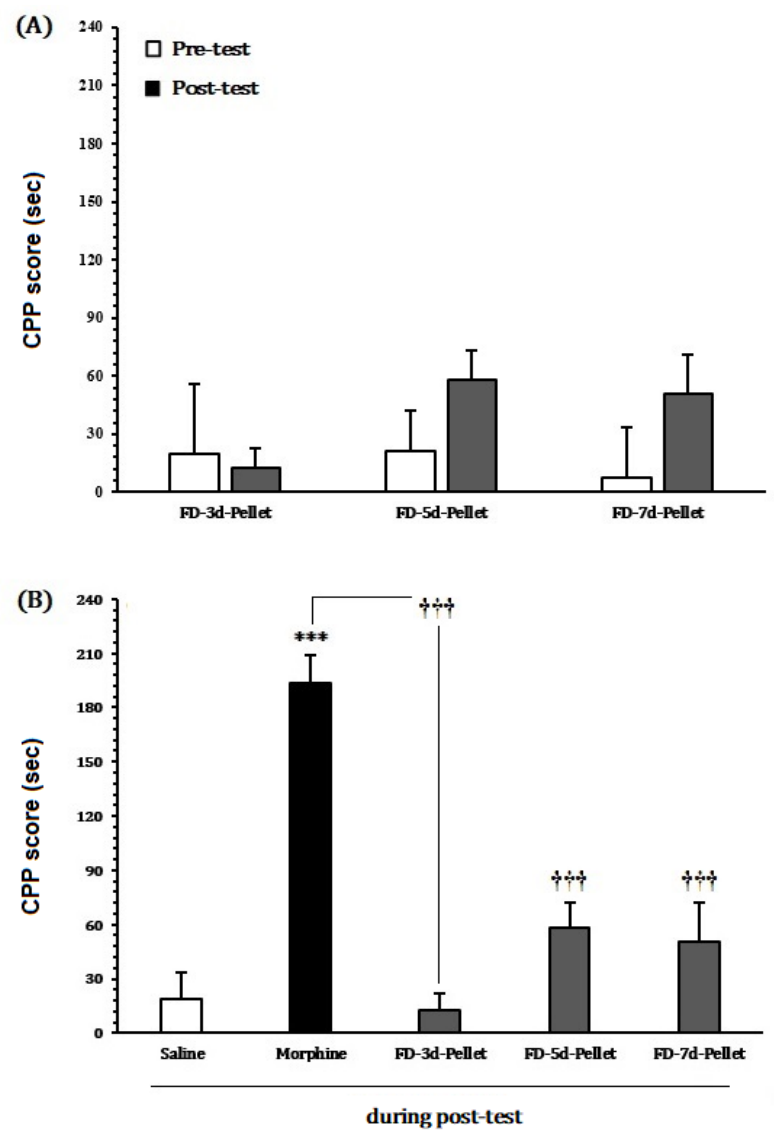

NEUROSCIENCE

Figure 4. A: CPP Scores of pre-test and post-test of deprived animals received pellet as reward during 3, 5, and 7 conditioning days, as FD-3d-Pellet, FD-5d-Pellet, and FD-7d-pellet, respectively; B: Comparing the CPP scores during the post-test phase between food-deprived animals and morphine-group

Data are presented as Mean \pm SEM

*** $\mathrm{P}<0.001$ compared with the pre-test.

${ }^{t+t} \mathrm{P}<0.001$ compared with the morphine group.

animals were weighted frequently until reaching $80 \%$ $85 \%$ of their initial weight $(15 \%-20 \%$ weight loss $)$. We showed the trend of weight loss in the food-restricted groups (Figure 3 ). The body-weight loss in each checkpoint $(0,2,4,6,8,10,12$, and 13) was calculated (in \%) compared to the initial checkpoint (W0) and this trend is presented in Figure 3. The two-way repeated-measures ANOVA, followed by Bonferroni multiple comparison test (treatment effect: F 5, 324=1.537, $\mathrm{P}=0.1939$; time effect: $\mathrm{F} 6,324=758.3, \mathrm{P}<0.001$; interaction effect: $\mathrm{F} 30$, $324=2.235, \mathrm{P}=0.003$ ), showed no significant difference between the \%weight loss (\%WL) of two consecutive checkpoints in each group and also, Figure 3 showed that the $15 \%-20 \%$ WL trend toward the final day (d13) of restriction compared to day $0(\mathrm{~d} 0)$ of the 6 restrictedgroups in terms of percentage. Also, results indicate no significant difference between any specific weight-loss checkpoint of each group compared to other groups.
Effect of treatment with morphine on the induction of reward

The paired $t$ test showed that the CPP score of animals treated with morphine $(5 \mathrm{mg} / \mathrm{kg}, \mathrm{SC})$ increased compared to the pre-test $(\mathrm{P}<0.001$; supplementary figure). These results showed that $5 \mathrm{mg} / \mathrm{kg}$ of morphine could induce a reward.

Effect of $24 \mathrm{~h}$ food deprivation on the induction of pellet reward and comparing the CPP score of food CPP and morphine CPP

To find out whether food deprivation can facilitate inducing the food reward (pellet as food reward), the animals experienced $24 \mathrm{~h}$ of food deprivation before the first day of the conditioning period while they had access to water. A paired $t$ test was used to examine the difference between the CPP scores of pre-test and post-test of each group: FD-3d-pellet, FD-5d-pellet, and FD-7d-pellet. 
The results showed no significant difference between the CPP score of pre-test and post-test of FD-3d-pellet $(\mathrm{P}=0.8629$; Figure 4A). Furthermore, no significant differences were found between pre-test and post-test of FD-5d-pellet ( $\mathrm{P}=0.0547$; Figure. $4 \mathrm{~A}$ ) and FD-7d-pellet group ( $\mathrm{P}=0.1378$; Figure $4 \mathrm{~A})$. Therefore, $24 \mathrm{~h}$ food deprivation did not facilitate the food CPP even though extinction of conditioning period ( 5 or 7 days).

One-way ANOVA followed by Tukey's multiple comparison tests $(\mathrm{P}<0.001$; Figure $4 \mathrm{~B})$ indicated a significant difference between the CPP scores of post-test of animals that received $5 \mathrm{mg} / \mathrm{kg}$ of morphine compared to the saline group $(\mathrm{P}<0.001)$ while there was no significant difference between the CPP scores of post-test of animals that experienced food deprivation and received food during 3, 5 and 7 days conditioning period compared to saline group. The CPP score of the morphine group was significantly different from the FD-3d-pellet, FD-5d-pellet, and FD-7d-pellet groups $(\mathrm{P}<0.001)$. These results showed the lack of food reward induction in deprived animals who experienced a different duration of conditioning days while receiving pellets as the reward.

Investigating CPP induced by biscuit or popcorn in food restricted rats

To determine whether food restriction can facilitate food reward, we restricted animals for food for two weeks before the conditioning period. Three different groups, FR-3d-B, FR-5d-B, and FR-7d-B, were conditioned in the CPP box with biscuits as food reward during the three, five, or seven days of conditioning period, respectively. The paired $t$ test indicated a significant difference in CPP scores between the pre-test and post-test of the FR-5d-B group $(\mathrm{P}<0.001$; Figure $5 \mathrm{~A})$ and FR-7d$\mathrm{B}$ group $(\mathrm{P}<0.001$; Figure $5 \mathrm{~A})$. However, there was no significant difference between the CPP score of pre-test and post-test of that group, which experienced a 3-day conditioning period ( $\mathrm{P}=0.3983$; Figure 5A). These data showed that biscuits as a palatable food could induce food (natural) reward during a 5- and 7-day conditioning period in the food-restricted animals.

The paired $t$ test showed a significant difference in the CPP score between pre-test and post-test in each group of animals that underwent two weeks of food restriction and was conditioned for 5 days $(\mathrm{P}<0.001$; Figure $5 \mathrm{~B})$ and 7 days $(\mathrm{P}<0.001$; Figure $5 \mathrm{~B})$ in the CPP box by popcorn as the food reward. On the other hand, there was no significant difference between pre-test and post-test of animals that experienced restriction and got popcorn during three days of conditioning $(\mathrm{P}=0.9251$; Figure
$5 B)$. These results indicated that popcorn could induce natural-CPP during 5-day and 7-day conditioning period in food-restricted animals.

Also, 2-way ANOVA, followed by Bonferroni's multiple comparison test (Treatment effect: F 1, 40=3.477 $\mathrm{P}=0.0770$; time effect: $\mathrm{F}_{2,40}=70.66 ; \mathrm{P}<0.001$; interaction effect: $\mathrm{F}_{2,40}=5.011 ; \mathrm{P}=0.0114$; Figure $\left.5 \mathrm{C}\right]$ showed a significant difference between the CPP score of restricted animals that received biscuit during a 7-day conditioning with those that received popcorn for 7 days $(\mathrm{P}<0.01)$. These results indicate that the potency of biscuits is more than popcorn in reward induction.

Comparing the CPP induced by palatable foods (biscuit or popcorn) with morphine CPP

One-way ANOVA followed by Tukey's multiple comparison tests $\left(\mathrm{F}_{4,39}=25.84 ; \mathrm{P}<0.001\right.$; Figure $\left.6 \mathrm{~A}\right)$ revealed that $\mathrm{CPP}$ scores of animals that received biscuits as a palatable food during 5- or 7-conditioning days (FR-5d$\mathrm{B}$ and FR-7d-B, respectively) increased compared to saline group. However, the CPP score of the FR-3d-B group did not increase compared to the control group, while there is a significant difference between this group and the morphine group $(\mathrm{P}<0.001)$. The CPP score of the group that received biscuits for 7 days, FR-7d-B, increased compared to the morphine group $(\mathrm{P}<0.01)$. These results indicate that biscuits as a food reward could induce reward in deprived animals in a durationdependent manner in terms of conditioning days.

To compare the CPP score of animals that got popcorn as the food reward with saline and morphine groups, we used 1-way ANOVA, followed by Tukey's multiple comparison test. The results $\left(\mathrm{F}_{4,38}=26.76\right.$; $\mathrm{P}<0.001$; Figure 6B) showed that the CPP score of animals that received popcorn for 5 or 7 days increased compared to the saline group $(\mathrm{P}<0.001)$. However, there is no significant difference between the CPP score of the group that received popcorn during 3 days of conditioning and the saline group. Therefore, these results indicate that popcorn could induce food reward in food-deprived animals.

3.6. The effect of morphine, food deprivation, and food restriction on locomotor activity

Furthermore, 1-way ANOVA, followed by Tukey's multiple comparison test $\left(\mathrm{F}_{10,74}=0.2781 ; \mathrm{P}=0.9842\right.$; Figure 7A), showed no statistically significant difference in the distance traveled among the groups. These results showed that neither morphine nor food deprivation or restriction affected locomotor activity among groups. 

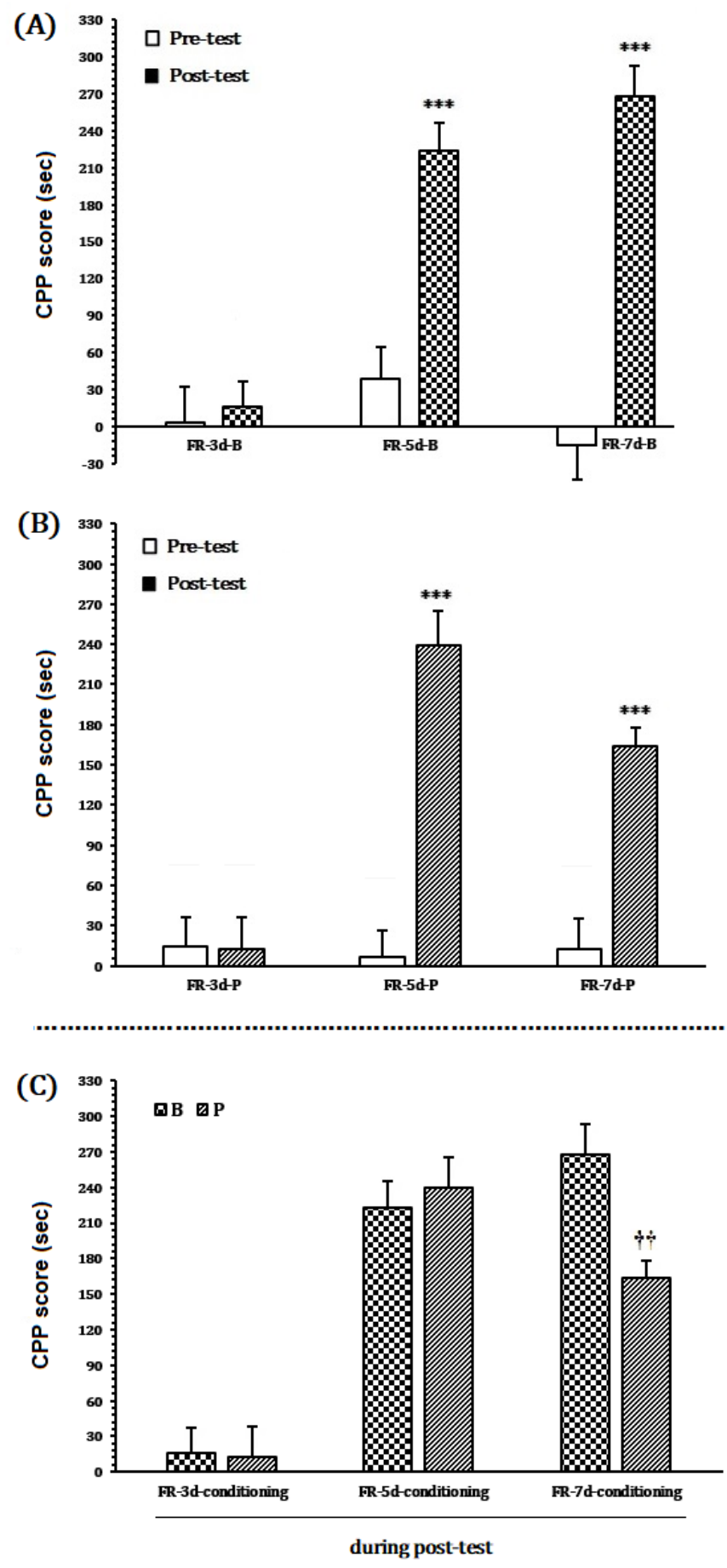

NEUR SCIENCE

Figure 5. A: Induction of food conditioned place preference (CPP) (comparing the cpp score of pre-test and post-test) in restricted animals received biscuit; $b$ : as reward during 3, 5, and 7 days of conditioning, as fr-3d-b, fr-5d-b, and fr-7d-b groups, respectively; B: comparing food-induced reward (cpp score as index) in restricted animals received popcorn (P) as food reward during 3, 5, and 7 days of conditioning, as fr-3d-p, fr-5d-p, and fr-7d-p groups, respectively; C: comparing the magnitude of reward induced between restricted animals received biscuit $(\mathrm{B})$ with popcorn $(\mathrm{P})$ in different conditioning periods $(3,5$, and 7 days).

Data are presented as Mean \pm SEM. *** $\mathrm{P}<0.001$ compared with the pre-test. $\dagger \dagger \mathrm{P}<0.01$ compared with the FR-7d-B group. 

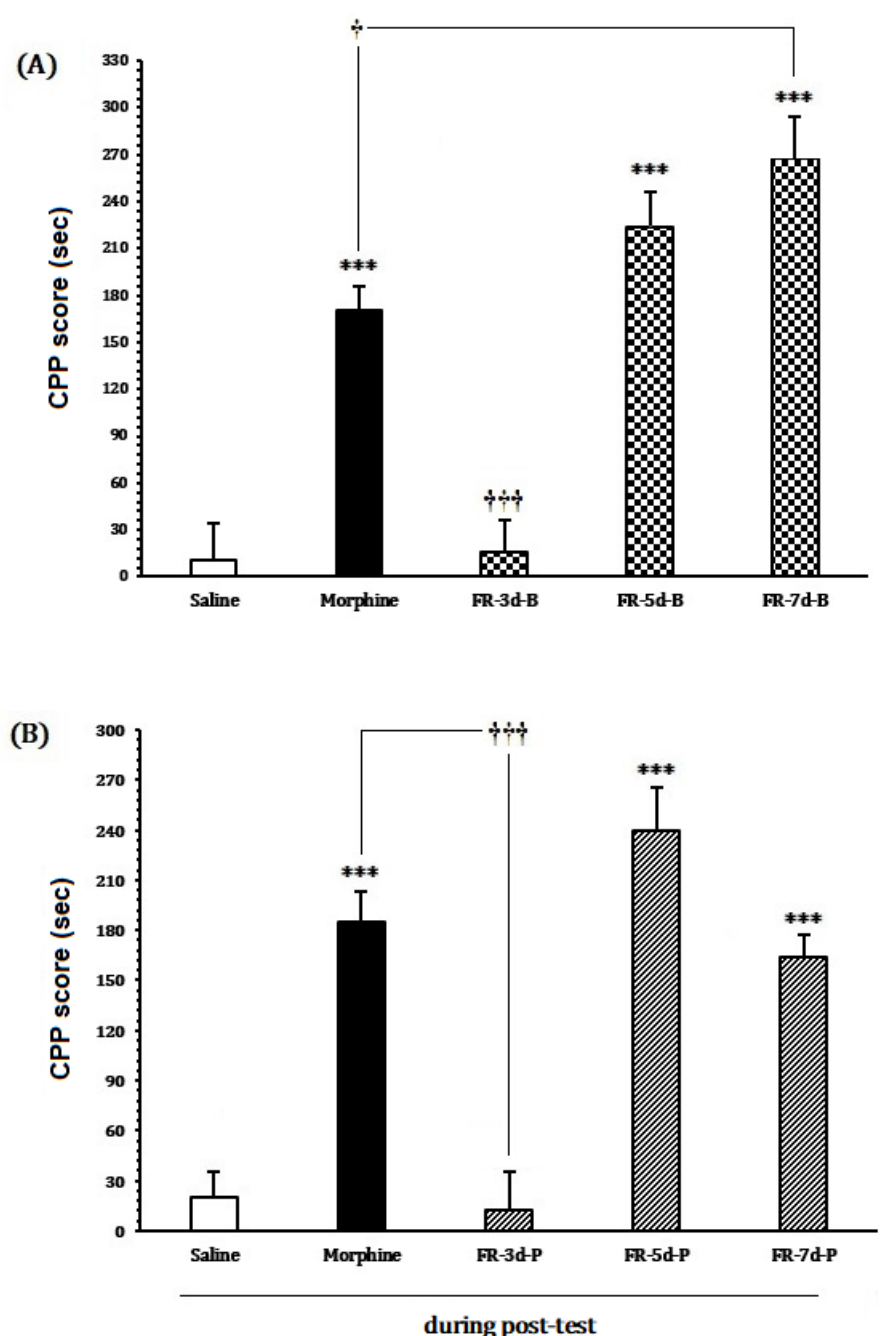

NEUR SCIENCE

Figure 6. A: Comparing the CPP scores during the post-test between food-restricted animals received biscuit during the acquisition phase for 3, 5, or 7 Days (FR-3d-B, FR-5d-B, or FR-7d-B groups) and morphine group; B: Comparing the CPP scores during post-test between food-restricted animals received popcorn during the acquisition Phase for 3, 5 or 7 days (FR-3d-P, FR-5d-P or FR-7d-P groups) and morphine group

Data are presented as Mean \pm SEM. *** $\mathrm{P}<0.001$ compared with the saline group. $\dagger \mathrm{P}<0.05$ and $\dagger+\dagger \mathrm{P}<0.001$ compared with the morphine group.

\section{Discussion}

To better treat addiction, which is a rising massive global problem, it is necessary to understand the mechanisms involved in the development and induction of reward-associated behaviors. In this regard, morphine, as an opioid, is one of the most important drugs of abuse. Moreover, food addiction is a growing trend that results in obesity, a current global issue due to the increased reward system activity. On the other hand, food intake assures the animal's survival, and food-induced reward, as a natural reward, is necessary for eating. Given this, manipulation of the reward system for controlling drug abuse disorder is a more criti- cal issue while facing addiction treatment, which can adversely influence natural rewards such as food and sex. Therefore, it is necessary to investigate different aspects of a drug (morphine) and natural reward (food) before considering an ideal treatment.

In this study, we aimed to explore and compare the reward-related behavior in morphine-induced and food-induced rewards (as a natural reward) in a CPP paradigm. At the same time, we examined different protocols for the induction of natural reward. 


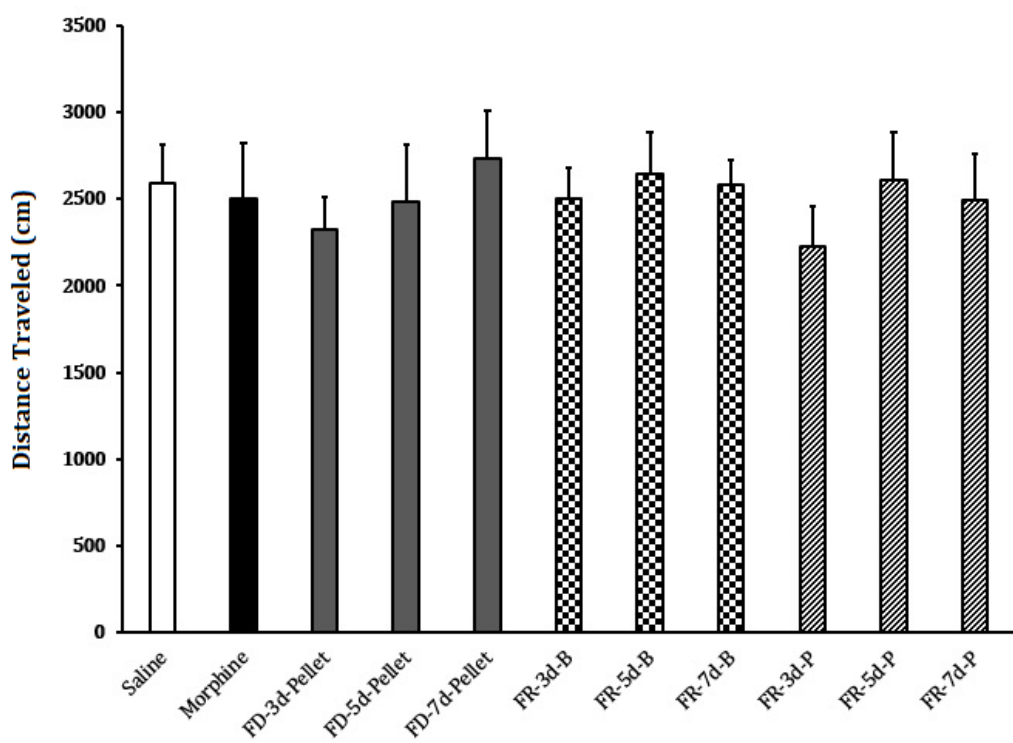

Figure 7. Distance travelled as the locomotor activity by all groups

The results show no significant difference between all groups. Data are presented as Mean \pm SEM.

The main findings of the present research demonstrated that 1) morphine ( $5 \mathrm{mg} / \mathrm{kg}$, SC) could induce morphine CPP; 2) the combination of food deprivation as a facilitator and pellet as a food reward, could not induce food CPP during 3, 5, and 7 days of conditioning (the food CPP was not induced in deprived animals which received pellet, as food reward during 3, 5, and 7 days of conditioning); 3 ) the food reward was induced in two groups of restricted animals that received biscuit during 5 or 7 days of conditioning while three days of conditioning could not induce food reward; 4) the food reward was induced in the two restricted animal groups that got popcorn during 5 or 7 days of conditioning; 5) the CPP scores of the restricted animals that received biscuit during seven days were higher than others that conditioned by popcorn during 7 days; 6) in the restricted group that experienced 7 days conditioning by biscuit the CPP score increased more than morphine; and 7) neither morphine nor FD/FR could affect locomotor activity.

Our results showed that food CPP was not induced in rats that experienced 24-h FD with multiple conditioning periods $(3,5$, and 7 days), while food restriction facilitated the food CPP in animals restricted from food and received palatable food during the acquisition phase. Contrary to the current study, Spiteri et al. showed that food deprivation $(24 \mathrm{~h})$ in mice could facilitate CPP to the pellet through an increase in the time spent in the conditioning compartment (Spiteri et al., 2000).
Our results showed that food reward was not induced in rats that experienced FD during multiple conditioning periods ( 3,5 , and 7 days). This difference may depend on the type of animals used in these studies (mice or rats, respectively). Previous studies show that sympathetic activation and glucocorticoid levels, as stress indexes, increased in the plasma of the rats exposed to FD for 24h (Heiderstadt, McLaughlin, Wrighe, Walker, \& Gomez-Sanchez, 2000). Therefore, FD may act as a stress factor and prevent the induction of reward in food-deprived animals. Also, other studies indicate that stress, 24-h FD, could mainly reduce food intake (Adam \& Epel, 2007). So, after 24h of food deprivation, animals will be hungry and additional food may not be necessary for maintaining homeostasis or health. Also, it seems that the first session of exposure to CPP (the first day of conditioning) can be a determining factor for an animal's behavior and conditioning of reward (Toth \& Gardiner, 2000). Therefore, the CPP scores did not significantly increase even when the conditioning days increased to 5 and 7 . Although studies use "deprivation" and "restriction" terms interchangeably, in our study, these procedures affect reward induction in different ways (Heiderstadt et al., 2000).

Contrary to the fact that food deprivation does not facilitate food CPP in response to regular food, food restriction facilitates food CPP in response to palatable food. To induce food reward, in the other subsets of our experiment, animals underwent food restriction. Studies show that food restriction augments the rewarding and motoractivating effects of direct DA receptor agonists, and the 
D1 dopamine antagonist reverses these induced effects of drugs. Also, food restriction increases the induction of c-Fos in limbic forebrain dopamine (DA) terminal areas in the presence of drug reward. Therefore, it seems that food restriction changes the dopamine level and receptor expression, and sensitivity in reward-related areas (Carr, 2007, 2020; Carr et al., 2000). Therefore, food deprivation and restriction may affect dopamine levels and the expression of dopamine receptors in reward-associated areas (Patterson et al., 1998; Raynor \& Epstein, 2003).

The observed increase of the CPP score in animals that received biscuits or popcorn as a reward following the extended duration of conditioning days $(3,5$, to 7 days $)$ showed a time-dependent manner of conditioning in the hedonic signals of eating.

The difference between the CPP score of animals that received biscuits during 7 days of conditioning and those that received popcorn shows that rats prefer biscuits more than popcorn under the same condition. Therefore, we concluded that the place preference observed after food conditioning is dependent on the reward-related behavior of feeding. The magnitude of food-induced reward is related to the palatability of foods (Khoo et al., 2018) as to their hedonic component (Coccurello \& Maccarrone, 2018; Harb et al., 2014). Also, the level of dopamine release in the nucleus accumbens (NAc), an important area of the reward system, increased in animals that received palatable food compared to regular chow (de Macedo et al., 2016). It is interesting that after the replacement of dopamine into NAc and putamen of dopamine-deficit animals (DA-/-), they started eating but showed interest only in palatable and sweet foods. Studies show that the dopaminergic system is activated in response to palatable food. Additionally, this system's activity is affected by modulators, such as orexin, ghrelin, and neuropeptide-Y (NPY).

The Ventral Tegmental Area (VTA) is known as the most critical dopaminergic region in the reward circuit that projects dopaminergic neurons to the amygdala (amyg), hippocampus (HIP), prefrontal cortex (PFC), NAc (Bernstein et al., 2018). The dopamine projections from amyg and PFC to the lateral hypothalamus $(\mathrm{LH})$ have a direct role in food intake (Kenny, 2011). Our results show that in animals conditioned by food and morphine, surprisingly, the CPP score of deprived animals that received biscuits during 7 days of conditioning was more than the morphine group. Lau et al. showed that naloxone could block morphine CPP and food CPP in zebrafish. Also, they showed that too few (tof) mutant zebrafish underwent reduction of a selection of dopaminergic and serotoninergic neurons in the basal dienceph- alon and were conditioned to food, while morphine did not induce any reward (Lau et al., 2006). So, these effects suggest that the regulation of morphine- and natural-rewardrelated behavior may recruit different pathways selectively targeted for the treatment of morphine abuse or addiction. Neuroplasticity of dopaminergic neurons of VTA is induced by exogenous opioids such as morphine. Also, endogenous opioid release during natural reward can induce neuroplasticity of these DA neurons using Mu-opioid receptors and $\beta$ endorphin. These results show the role of the plasticity of VTA-DA neurons in natural and morphine reward memory (Pitchers et al., 2014).

Studies show that food reward induces cortical acetylcholine release that requires orexin signaling through the OX1 receptor. In response to food reward, the projections from accumbens and amyg affect LHorexin neurons, which play an important role in food CPP (Zheng et al., 2007). Fos-expression in NAc shell, $\mathrm{LH}$, and basolateral amygdala decreased when abstinent animals experienced food-CPP, while it increased in animals tested for morphine CPP (Aston-Jones \& Harris, 2004).

\section{Conclusion}

In conclusion, our results show that palatable food could induce reward and morphine. Moreover, food restriction is a better facilitator than food deprivation for the induction of food reward. Therefore, in future studies, we can consider food-induced reward as a natural reward compared to morphine reward for electrophysiological and molecular investigation.

\section{Ethical Considerations}

\section{Compliance with ethical guidelines}

Experimental procedures were approved by the Ethics Committee of Shahid Beheshti University of Medical Sciences (IR.SBMU.SM.REC.1395.373), Tehran, Iran. All protocols followed the internationally accepted principles for the Care and Use of Laboratory Animals (National Institutes of Health [NIH] publication No. 80-23, revised in 1996).

\section{Funding}

Funding for this study was provided by the Neuroscience Research Center, School of Medicine, Shahid Beheshti University of Medical Sciences (Grant No.: 373 ). The Neuroscience Research Center had no further role in the design of the study; in the collection, analysis and 
interpretation of data; in the writing of the report; and in the decision to submit the paper for publication. This article has been extracted from the thesis written by Shole Jamali in the Neuroscience Research Center, School of Medicine, Shahid Beheshti University of Medical Sciences (Grant/Registration No.: 373).

\section{Authors' contributions}

All authors equally contributed to preparing this article.

\section{Conflict of interest}

The authors declared no conflict of interest.

\section{References}

Adam, T. C., \& Epel, E. S. (2007). Stress, eating and the reward system. Physiology \& Behavior, 91(4), 449-458. [DOI:10.1016/j. physbeh.2007.04.011] [PMID]

Allen, P. J., Batra, P., Geiger, B. M., Wommack, T., Gilhooly, C., \& Pothos, E. N. (2012). Rationale and consequences of reclassifying obesity as an addictive disorder: Neurobiology, food environment and social policy perspectives. Physiology \& Behavior, 107(1), 126-137. [PMID] [PMCID]

Aston-Jones, G., \& Harris, G. C. (2004). Brain substrates for increased drug seeking during protracted withdrawal. Neuropharmacology, 47, (Suppl 1), 167-179. [PMID]

Avena, N. M., Rada, P., \& Hoebel, B. G. (2008). Evidence for sugar addiction: Behavioral and neurochemical effects of intermittent, excessive sugar intake. Neuroscience $\mathcal{E}$ Biobehavioral Reviews, 32(1), 20-39. [PMID] [PMCID]

Bernstein, D. L., Badve, P. S., Barson, J. R., Bass, C. E., \& España, R. A. (2018). Hypocretin receptor 1 knockdown in the ventral tegmental area attenuates mesolimbic dopamine signaling and reduces motivation for cocaine. Addiction Biology, 23(5), 1032-1045. [PMID] [PMCID]

Carlini, V. P., Martini, A. C., Schiöth, H. B., Ruiz, R. D., Fiol de Cuneo, M., \& de Barioglio, S. R. (2008). Decreased memory for novel object recognition in chronically food-restricted mice is reversed by acute ghrelin administration. Neuroscience, 153(4), 929-934. [PMID]

Carr, K. D. (2007). Chronic food restriction: Enhancing effects on drug reward and striatal cell signaling. Physiology $\&$ Behavior, 91(5), 459-472. [PMID]

Carr, K. D. (2020). Modulatory effects of food restriction on brain and behavioral effects of abused drugs. Current Pharmaceutical Design, 26(20), 2363-2371. [PMID] [PMCID]

Carr, K. D., Kim, G. Y., \& Cabeza de Vaca, S. (2000). Chronic food restriction in rats augments the central rewarding effect of cocaine and the delta1 opioid agonist, DPDPE, but not the delta2 agonist, deltorphin-II. Psychopharmacology, 152(2), 200-207. [PMID]
Coccurello, R., \& Maccarrone, M. (2018). Hedonic eating and the "delicious circle": From lipid-derived mediators to brain dopamine and back. Frontiers in Neuroscience, 12, 271. [PMID]

Conrad, C. D. (2010). A critical review of chronic stress effects on spatial learning and memory. Progress in Neuro-Psychopharmacology and Biological Psychiatry, 34(5), 742-755. [PMID]

de Macedo, I. C., de Freitas, J. S., \& da Silva Torres, I. L. (2016). The influence of palatable diets in reward system activation: A mini review. Advances in Pharmacological Sciences, 2016, 7238679. [PMID] [PMCID]

Cabeza de Vaca, S., \& Carr, K. D. (1998). Food restriction enhances the central rewarding effect of abused drugs. The Journal of Neuroscience: The Official Journal of The Society for Neuroscience, 18(18), 7502-7510. [PMCID]

Domingo-Rodriguez, L., Ruiz de Azua, I., Dominguez, E., Senabre, E., Serra, I., \& Kummer, S., et al. (2020). A specific prelimbic-nucleus accumbens pathway controls resilience versus vulnerability to food addiction. Nature Communications, 11(1) 782. [DOI:10.1038/s41467-020-14458-y] [PMID] [PMCID]

Duan, Y., Shen, F., Gu, T., \& Sui, N. (2016). Addiction: From context-induced hedonia to appetite, based on transition of micro-behaviors in morphine abstinent tree shrews. Frontiers in Psychology, 7, 816.[DOI:10.3389/fpsyg.2016.00816]

Gold, M. S., Graham, N. A., Cocores, J. A., \& Nixon, S. J. (2009). Food addiction? Journal of Addiction Medicine, 3(1), 42-45. [PMID]

Grosshans, M., Loeber, S., \& Kiefer, F. (2011). Implications from addiction research towards the understanding and treatment of obesity. Addiction Biology, 16(2), 189-198. [PMID]

Harb, M. R., Sousa, N., Zihl, J., \& Almeida, O. F. (2014). Reward components of feeding behavior are preserved during mouse aging. Frontiers in Aging Neuroscience, 6, 242. [PMID] [PMCID]

Heiderstadt, K. M., McLaughlin, R. M., Wright, D. C., Walker, S. E., \& Gomez-Sanchez, C. E. (2000). The effect of chronic food and water restriction on open-field behaviour and serum corticosterone levels in rats. Laboratory Animals, 34(1), 20-28. [PMID]

Hogarth L. (2020). Addiction is driven by excessive goaldirected drug choice under negative affect: Translational critique of habit and compulsion theory. Neuropsychophar macology: Official Publication of the American College of Neuropsychopharmacology, 45(5), 720-735. [PMID] [PMCID]

IInbar, D., Gendelis, S., Mesner, S., Menahem, S., \& Kupchik Y. M. (2020). Chronic calorie-dense diet drives differences in motivated food seeking between obesity-prone and resistant mice. Addiction Biology, 25(3), e12753. [PMID]

Kenny, P. J. (2011). Common cellular and molecular mechanisms in obesity and drug addiction. Nature Reviews Neuroscience, 12(11), 638-651. [DOI:10.1038/nrn3105] [PMID]

Khoo, S. Y., Clemens, K. J., \& McNally, G. P. (2018). Palatable food self-administration and reinstatement are not affected by dual orexin receptor antagonism. Progress in Neuro-Psychopharmacology and Biological Psychiatry, 87(Pt A), 147-157. [DOI:10.1016/j.pnpbp.2017.06.028] [PMID] 
Krashes, M. J., \& Kravitz, A. V. (2014). Optogenetic and chemogenetic insights into the food addiction hypothesis. Frontiers in Behavioral Neuroscience, 8, 57. [PMID] [PMCID]

Lau, B., Bretaud, S., Huang, Y., Lin, E., \& Guo, S. (2006). Dissociation of food and opiate preference by a genetic mutation in zebrafish. Genes, Brain and Behavior, 5(7), 497-505. [PMID]

Lee, K., Claar, L. D., Hachisuka, A., Bakhurin, K. I., Nguyen, J., \& Trott, J. M., et al. (2020). Temporally restricted dopaminergic control of reward-conditioned movements. $\mathrm{Na}$ ture Neuroscience, 23(2), 209-216. [PMID] [PMCID]

Leigh, S. J., \& Morris, M. J. (2018). The role of reward circuitry and food addiction in the obesity epidemic: An update. Biological Psychology, 131, 31-42. [PMID]

Lew-Starowicz, M., Lewczuk, K., Nowakowska, I., Kraus, S., \& Gola, M. (2020). Compulsive sexual behavior and dysregulation of emotion. Sexual Medicine Reviews, 8(2), 191-205. [PMID]

Marion-Poll, L., Besnard, A., Longueville, S., Valjent, E., Engmann, O., \& Caboche, J., et al. (2019). Cocaine conditioned place preference: unexpected suppression of preference due to testing combined with strong conditioning. Addiction Biology, 24(3), 364-375. [PMID]

Ng, T. H., Alloy, L. B., \& Smith, D. V. (2019). Meta-analysis of reward processing in major depressive disorder reveals distinct abnormalities within the reward circuit. Translational Psychiatry, 9(1), 293. [DOI:10.1038/s41398-019-0644-x] [PMID] [PMCID]

Olsen, C. M. (2011). Natural rewards, neuroplasticity, and non-drug addictions. Neuropharmacology, 61(7), 1109-1122. [PMID] [PMCID]

Ong, Z. Y., \& Muhlhausler, B. S. (2011). Maternal "junk-food" feeding of rat dams alters food choices and development of the mesolimbic reward pathway in the offspring. FASEB Journal: Official Publication of The Federation of American Societies for Experimental Biology, 25(7), 2167-2179. [PMID] [PMCID]

Ouyang, J., Carcea, I., Schiavo, J. K., Jones, K. T., Rabinowitsch, A., \& Kolaric, R., et al. (2017). Food restriction induces synaptic incorporation of calcium-permeable AMPA receptors in nucleus accumbens. The European Journal of Neuroscience, 45(6), 826-836. [PMID] [PMCID]

Patterson, T. A., Brot, M. D., Zavosh, A., Schenk, J. O., Szot, P., \& Figlewicz, D. P. (1998). Food deprivation decreases mRNA and activity of the rat dopamine transporter. Neuroendocrinology, 68(1), 11-20. [PMID]

Pitchers, K. K., Coppens, C. M., Beloate, L. N., Fuller, J., Van, S., \& Frohmader, K. S., et al. (2014). Endogenous opioid-induced neuroplasticity of dopaminergic neurons in the ventral tegmental area influences natural and opiate reward. The Journal of Neuroscience : The Official Journal of The Society for Neuroscience, 34(26), 8825-8836. [PMID] [PMCID]

Pourhamzeh, M., Mozafari, R., Jamali, S., Motamedi, F., Ahadi, R., \& Haghparast, A. (2019). Involvement of orexin receptors within the hippocampal dentate gyrus in morphine-induced reinstatement in food-deprived rats. Behavioural Brain Research, 375, 112155. [PMID]
Prescott, M. J., Brown, V. J., Flecknell, P. A., Gaffan, D., Garrod, K., \& Lemon, R. N., et al. (2010). Refinement of the use of food and fluid control as motivational tools for macaques used in behavioural neuroscience research: Report of a Working Group of the NC3Rs. Journal of Neuroscience Methods, 193(2), 167-188. [PMID]

Raynor, H. A., \& Epstein, L. H. (2003). The relative-reinforcing value of food under differing levels of food deprivation and restriction. Appetite, 40(1), 15-24. [DOI:10.1016/S01956663(02)00161-7

Rosenblum, A., Marsch, L. A., Joseph, H., \& Portenoy, R. K. (2008). Opioids and the treatment of chronic pain: controversies, current status, and future directions. Experimental and Clinical Psychopharmacology, 16(5), 405-416. [PMID] [PMCID]

Russo, S. J., \& Nestler, E. J. (2013). The brain reward circuitry in mood disorders. Nature Reviews Neuroscience, 14(9), 609 625. [DOI:10.1038/nrn3381] [PMID] [PMCID]

Sampey, B. P., Vanhoose, A. M., Winfield, H. M., Freemerman, A. J., Muehlbauer, M. J., \& Fueger, P. T., et al. (2011). Cafeteria diet is a robust model of human metabolic syndrome with liver and adipose inflammation: Comparison to high-fat diet. Obesity, 19(6), 1109-1117. [PMID] [PMCID]

Saper, C. B., Chou, T. C., \& Elmquist, J. K. (2002). The need to feed: Homeostatic and hedonic control of eating. Neuron, 36(2), 199-211. [PMID]

Shirazy, M., RayatSanati, K., Jamali, S., Motamedi, F., \& Haghparast, A. (2020). Role of orexinergic receptors in the dentate gyrus of the hippocampus in the acquisition and expression of morphine-induced conditioned place preference in rats. Behavioural Brain Research, 379, 112349. [PMID]

Spiteri, T., Le Pape, G., \& Ågmo, A. (2000). What is learned during place preference conditioning? A comparison of food-and morphine-induced reward. Psychobiology, 28(3), 367-382. [DOI:10.3758/BF03331994]

Stice, E., Figlewicz, D. P., Gosnell, B. A., Levine, A. S., \& Pratt, W. E. (2013). The contribution of brain reward circuits to the obesity epidemic. Neuroscience \& Biobehavioral Reviews, 37(9 Pt A), 2047-2058. [PMID]

Strang, J., Volkow, N. D., Degenhardt, L., Hickman, M., Johnson, K., \& Koob, G. F., et al. (2020). Opioid use disorder. Nature reviews. Disease Primers, 6(1), 3. [PMID]

Toth, L. A., \& Gardiner, T. W. (2000). Food and water restriction protocols: Physiological and behavioral considerations. Contemporary Topics in Laboratory Animal Science, 39(6), 9-17. [PMID]

Vallöf, D., Kalafateli, A. L., \& Jerlhag, E. (2020). Brain regionspecific neuromedin $\mathrm{U}$ signalling regulates alcohol-related behaviours and food intake in rodents. Addiction Biology, 25(3), e12764. [PMID] [PMCID]

Williams, D. L., Coiduras, I. I., Parise, E. M., \& Maske, C. B. (2020). Hindbrain orexin 1 receptors blunt intake suppression by gastrointestinal nutrients and cholecystokinin in male rats. Peptides, 133, 170351. [PMID] [PMCID]

Zheng, H., Patterson, L. M., \& Berthoud, H. R. (2007). Orexin signaling in the ventral tegmental area is required for highfat appetite induced by opioid stimulation of the nucleus accumbens. The Journal of Neuroscience: The Official Journal of The Society for Neuroscience, 27(41), 11075-11082. [PMID] [PMCID] 